\title{
2 Unraveling the Performance of Dispersion-Corrected 3 Functionals for the Accurate Description of Weakly 4 Bound Natural Polyphenols
}

${ }^{1}$ Division of Theoretical Chemistry, Department of Physics, Chemistry and Biology (IFM),

7 Linköping University, SE-58183 Linköping, Sweden

$8{ }^{2}$ Laboratoire de Chimie des Substances Naturelles, Université de Limoges, 2 rue du Dr

9 Marcland, F-87025 Limoges $C E D E X$, France

$10{ }^{3}$ INSERM UMR 850, Univ. Limoges, Faculté de Pharmacie, 2 rue du Dr Marcland, F-87025

11 Limoges, France

$12{ }^{4}$ Regional Centre of Advanced Technologies and Materials, Department of Physical Chemistry, 13 Faculty of Science, Palacký University of Olomouc, tr. 17 listopadu, 77146 Olomouc, Czech 14 Republic

$15{ }^{5}$ Departamento de Química Física, Universidad de Alicante, Apartado de Correos 99, E-03080, 16 Alicante, Spain

* Corresponding author: Florent Di Meo, flodi20@ifm.liu.se 


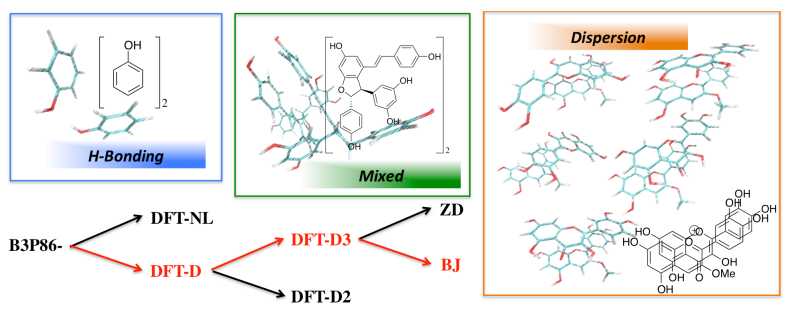

\section{ABSTRACT}

4 Long-range non-covalent interactions play a key role in the chemistry of natural polyphenols.

5 We have previously proposed a description of supramolecular polyphenol complexes by the

6 B3P86 density functional coupled with some corrections for dispersion. We couple here the

7 B3P86 functional with the D3 correction for dispersion, assessing systematically the accuracy of

8 the new B3P86-D3 model using for that the well-known S66, HB23, NCCE31 and S12L datasets

9 for non-covalent interactions. Furthermore, the association energies of these complexes were

10 carefully compared to those obtained by other dispersion-corrected functionals, such as

11 B(3)LYP-D3, BP86-D3 or B3P86-NL. Finally, all this set of models were also applied to a

12 database composed of seven non-covalent polyphenol complexes of the most interest. 


\section{Introduction}

Polyphenols constitute one of the most important groups of natural compounds with ca. $10^{5}$ defined structures including phenolic acids, lignans, flavonoids, coumarins and stilbenoids. They are abundant in the plant kingdom and can be isolated from all plant organs (e.g., wood, root, flower, leaf, fruit) [1,2]. Their properties have been extensively investigated over the past years. First of all, because the human diet is rich in polyphenols and due to their chemical diversity, they exhibit a broad range of biological activities including cardiovascular [3], hepatic [4,5] and neurologic [6] protective activities. Second, elucidation of their biomimetic synthesis has always been a very active field of research [7] with the purpose of discovering new active compounds. Last but not the least, numerous polyphenols are colored (e.g., anthocyanidins, chalconoids, curcuminoids), which opens many opportunities in food industry to control pigmentation and develop new hues.

Theoretical chemistry has provided useful insights for all this phenomena by giving an accurate atomistic-like picture for the understanding of their macroscopical activities [8-15]. It has been of particular help to rationalize the capacity of polyphenols to form non-covalent selfassemblies in real-world samples. Many physical chemical processes are influenced by these supramolecular assemblies such as (i) UV/Vis properties [16,15,17] and thus pigmentation, (ii) regio- and stereoselectivity of oxidative coupling [18-20,14], and (iii) kinetics of free radical scavenging [12]. The related key biological processes strongly depend on the underlying noncovalent interactions such as: (i) plant color persistence [16,13] and hue variety in fruit and beverages [21,22], (ii) biomimetic syntheses [7], and (iii) antioxidant activity [12,23,24], respectively. These fields now require more than ever a deep understanding at the molecular level, which can be efficiently supported by quantum-chemical calculations. The cost-efficient yet accurate treatment of these interactions by ab initio methods is still challenging regarding the large size of these real-world systems and the interplay between electrostatic and dispersion interactions. Thus, Density Functional Theory (DFT) nowadays has become a judicious compromise to perform such calculations for large systems, reaching a high accuracy at a reasonable computational time. On one hand, the use of fine-tuned expressions for non-covalent interactions, such as dispersion-corrected DFT-D [25-31] or DFT-NL [32-34] methods, is key to accurately describe supramolecular assemblies of natural compounds. The choice of the ad equate functional is, on the other hand, also important for the description of electronic structure 
1 issues. In this context, we have recently proposed the coupling of the B3P86 functional with the

2 DFT-D2 [15] and DFT-NL formalisms [14], since B3P86 has been shown to behave accurately

3 enough for the rich chemistry of polyphenol compounds [15,14,35-37]. Therefore, the present

4 study aims at an improvement of this description using the DFT-D3 formalism and then the corresponding B3P86-D3 method is thoroughly assessed against standard non-covalent databases, but also for a 7-member group of non-covalent natural polyphenol systems including selected supramolecular geometries $[38,15,14]$ of the most interest (see Scheme 1 and Figure 1).

(a)

(b)<smiles>Oc1ccccc1</smiles>

\section{9} polyphenol systems<smiles>Oc1ccc(/C=C/c2cc(O)cc3c2[C@@H](c2cc(O)cc(O)c2)[C@@H](c2ccc(O)cc2)O3)cc1</smiles>

(c)<smiles>COc1cc2c(O)cc(O)cc2[o+]c1-c1ccc(O)c(O)c1</smiles>

Scheme 1. Chemical structures of (a) phenol, (b) $\varepsilon$-viniferin, (c) 3-O-methylcyanidin and (d) quercetin. The hydrogen atoms and corresponding $\mathrm{C}-\mathrm{H}$ bonds have been omitted for clarity.

To do it, the parameters entering into the -D3 correction have been fitted against the S66 database following a brute-force procedure. It must be stressed that two types of damping functions have been proposed within the DFT-D formalism, namely Zero-Damping (ZD) and Becke-Johnson[39-41] (BJ) damping functions. To evaluate the robustness of the new model, both B3P86-D3(BJ) and B3P86-D3(ZD) functionals were first tested against the S66 [42], HB23 [43] and NCCE31 [44-46] databases and consequently compared to well-known Becke family functionals, i.e., BP86, BLYP, B3LYP and B3P86 variants (including or not various dispersion corrections). Our previous parameterizations of B3P86-D2 and B3P86-NL are also included to underline the improvement provided by DFT-D3 formalism. Finally, the B3P86-D3 performance is validated on more real-world systems from S12L database [47] and seven natural non-covalent 


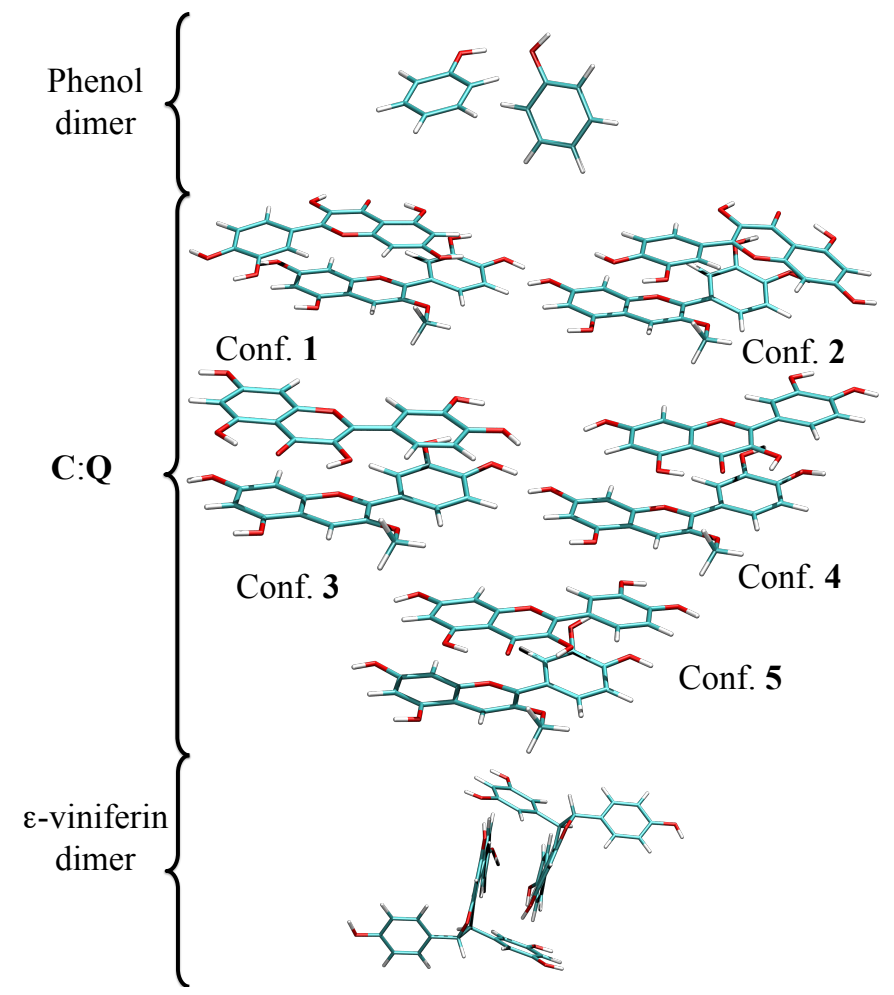

Figure 1. Optimized structures of the 7-member group of non-covalent natural polyphenol systems. The corresponding XYZ Coordinates are available in Supporting Information.

\section{Theoretical considerations}

\subsection{Computational details}

All calculations were performed with the ORCA 2.8.1 and 2.9.0 packages for both DFT-D2 and DFT-NL formalisms, respectively [48], while the DFT-D3 study (i.e., ZD- and BJ-functions as well as three-body term) was carried out using Grimme's code [49].

The different parameters for the -D3 function were obtained by a brute-force method for both ZD and BJ functions, namely B3P86-D3(ZD) and B3P86-D3(BJ), respectively. With the former function, a set of $s_{8}$ and $s_{r, 6}$ values in the ranges 0.500-3.500 and 0.500-2.100, respectively, were tested against the S66 dataset. For the latter function, the $s_{8}, \alpha_{1}$ and $\alpha_{2}$ values were tested in the 0.700-3.000, 0.300-0.700 and 4.000-5.000 ranges, respectively. This screen will cover 
extensively all the physically meaningful space (vide infra). It must be stressed that the elucidation of B3P86-D3(ZD) parameters was investigated in order to provide a complete assessment of B3P86-D3 refinement. However, the use of ZD function is not recommended with respect to BJ one since the latter consider the correct physical short-range behavior.[Ref]

The Def2-QZVPP (large) basis set used is believed to be close to the asymptotic region, thus preventing from Basis Set Superposition Error (BSSE) [14]. Note also that we used Resolutionof-the-Identity (RI) together with the "Chain-Of-Sphere eXchange" (COSX) algorithms, leading to dramatic speed-up of calculations without exhibiting any significant lack of accuracy (see Supporting information) [50,51]. The Def2-QZVPP auxiliary basis set (i.e., Def2-QZVPP/JK) was taken from the hardwired library. Numerical thresholds were systematically increased during the B3P86-D3 parameterization (i.e., VeryTightSCF, NoFinalGrid, GRID7, GRIDX6) with respect to the defaults to avoid as much as possible any numerical error. The numerical threshold and basis set were slightly modified for both S12L and polyphenol datasets to overcome numerical stability issues on very large systems (i.e., Def2-TZVPP, TightSCF, NoFinalGrid, GRID6, GRIDX5). When needed (i.e., polyphenol complexation measured in water [52]), calculations were performed using implicit solvent models in which the solute is embedded in a shape-adapted cavity surrounded by a structure-less dielectric continuum, namely COnductorlike Screening MOdel (COSMO) [53].

\subsection{Comparison of B3P86-D3 parameters with respect to known functionals}

A true minimum in the three-parameter dependent hypersurface was obtained with $s_{8}=2.483, \alpha_{1}$ $=0.541$ and $\alpha_{2}=4.306$ for B3P86-D3(BJ). As expected, $s_{8}$ is $24 \%$ higher for the GGA functional compared to the corresponding hybrid $\left(s_{8}=3.282\right.$ and 2.483 for BP86-D3(BJ) and B3P86D3(BJ), respectively, see Supporting Information). This is similarly observed for the BLYPD3(BJ)/B3LYP-D3(BJ) values ( $s_{8}$ is 26\% higher for BLYP-D3(BJ)). This trend is in perfect agreement with previous studies since B88-based exchange functionals have been shown to exhibit a larger $s_{8}$ parameter than others (e.g., PBE0-D3(BJ) $s_{8}$ parameter is 1.218) [28]. Besides, $\alpha_{I}\left(\alpha_{2}\right)$ parameters are higher(lower) for B3P86-D3(BJ) than for B3LYP-D3(BJ), while it is higher(lower) for BLYP-D3(BJ) than for BP86-D3(BJ) (see Table 1a). This might indicate how the correction of B3P86-D3(BJ) is less long-ranged and more atom-pair dependent than B3LYP- 
1 D3(BJ). In other words, B3P86 inherently requires less correction because behaves less repulsively than B3LYP, which also agrees with the previous DFT-D2 parameterization since $s_{6}$ was also found to be lower for B3P86-D2 than for B3LYP-D2 [15].

The two functional-dependent parameters for B3P86-D3(ZD) have been also correspondingly obtained ( $s_{8}=0.720$ and $s_{r, 6}=1.110$, see Supporting Information). The value of $s_{8}$ for B3P86D3(ZD) is significantly lower than for B3LYP-D3(ZD) ( $s_{8}=0.720$ and 1.703 for B3P86-D3(ZD) and B3LYP-D3(ZD), respectively, see Supporting Information).

As a first sight, the combination of the hybrid B88 exchange functional with the P86 correlation functional might better perform for long-range description than the combination with LYP as a correlation functional. It must be noted that BLYP has appeared less repulsive than BP86 [27], in contradiction with the present results obtained for the hybrid forms. Our results clearly suggest that the hybrid form B3P86 might be well-suited for an accurate description of long-range interactions when coupled with a correction for dispersion. We hope to confirm these finding next by assessing the B3P86-D3 parameterizations against various databases dedicated to non-covalent interactions.

\section{Relevance of B3P86-D3 against standard databases}

\subsection{The biochemically inspired S66 database}

The S66 database comprises a set of bioinspired molecular structures (e.g., amino acids from proteins or nucleic acids from DNA) exhibiting the most common non-covalent interactions (including dispersion, electrostatic and mixed systems) [42]. A summary of the benchmarking done is presented in Figure 2a with further details for the different subsets reported in Table 1. MAD was reduced to 0.46 and $0.36 \mathrm{kcal}^{\mathrm{mol}}{ }^{-1}$ for B3P86-D2 and B3P86-NL, respectively (see Figure 2a), thus validating the previous parameterization of B3P86 within the DFT-D2 and DFTNL framework using smaller databases for it [15,14]. Interestingly, the MAD of B3P86-D3 is ca. halved with respect to B3P86-D2, whatever the damping function is used (MAD $=0.24$, kcal.mol ${ }^{-1}$ for both B3P86-D3(BJ) and -D3(ZD), see Figure 2a).

Broadly speaking, B3P86-D3 results are slightly better than BP86-D3 and B3LYP-D3 but without outperforming those obtained by BLYP-D3 (e.g., MAD $=0.31,0.25$ and $0.17 \mathrm{kcal}^{\mathrm{mol}}{ }^{-1}$,

\section{Florent Di Meo 21/9/2015 13:22}

Deleted: The uncorrected B3P86 exhibits the lowest MAD $\left(2.93 \mathrm{kcal}^{\mathrm{mol}}{ }^{-1}\right)$ with respect to uncorrected BLYP, BP86 and B3LYP (MAD $=4.07$, 3.60 , and $3.25 \mathrm{kcal}^{\mathrm{mol}} \mathrm{m}^{-1}$, respectively, see Figure

2a). 
1 for BP86-D3, B3LYP-D3 and BLYP-D3, respectively, see Figure 2a). However, no clear

2 statement can be provided regarding the slight differences observed between these functionals.

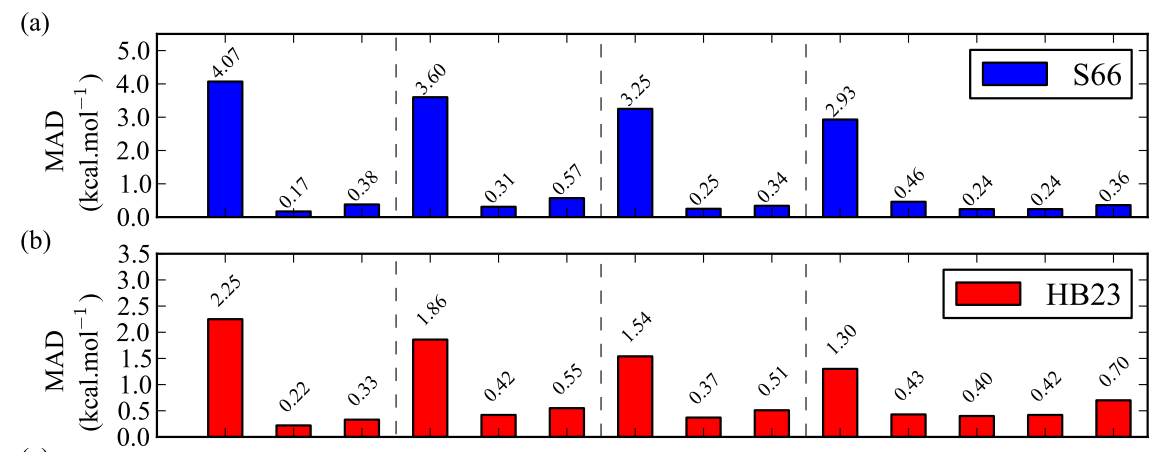

(c)

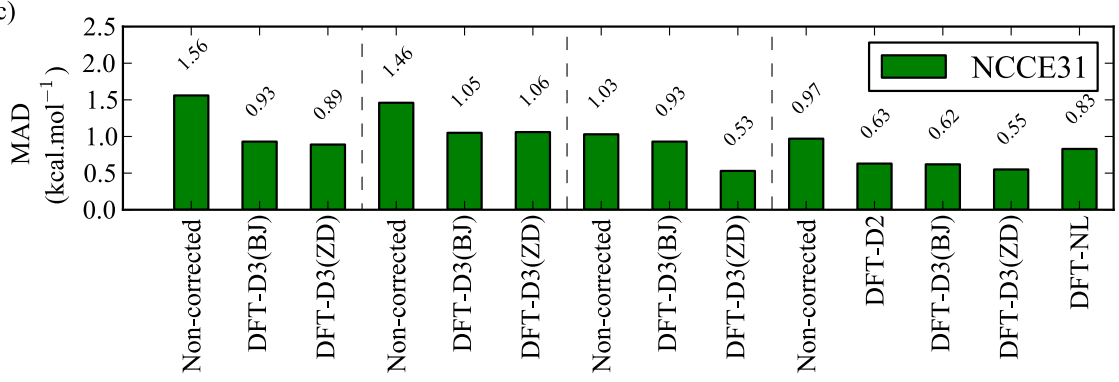

3

BLYP

BP86

B3LYP

B3P86

Figure 2. Mean absolute deviation (MAD, $\mathrm{kcal}^{\mathrm{mol}}{ }^{-1}$ ) of selected functionals with or without dispersion corrections, for the (a) total S66, (b) HB23 and (c) NCCE31 benchmarks. All the calculations were performed with the Def2-QZVPP basis set.

In the remaining of this section, only the dispersion and mixed (electrostatic and dispersion) interactions are separately discussed, as the S66 electrostatic-like subset is contained (vide infra) in the HB23 database. Among all the functionals used, the lowest MAD values for the S66 dispersion subset are again in a very narrow range. Actually, the lowest MAD is observed for both B3P86-D3 revisions $\left(\mathrm{MAD}=0.15 \mathrm{kcal}^{\mathrm{mol}}{ }^{-1}\right.$ for both B3P86-D3(BJ) and -D3(ZD), see Table 1). It must be emphasized that B3P86-NL seems also suitable for dispersion-driven systems while B3P86-D2 and B3LYP-D3(ZD) appears less appropriate (MAD = 0.17, 0.66 and 
$10.29 \mathrm{kcal}^{\mathrm{mol}}{ }^{-1}$, for B3P86-NL, -D2 and B3LYP-D3(ZD), respectively - see Table 1). On the 2 other hand, no significant difference is observed between the different corrections for the mixed 3 systems (e.g., MAD = 0.26 and $0.13 \mathrm{kcal} \mathrm{mol}^{-1}$ for B3P86-D2 and B3P86-D3(BJ), respectively, 4 Table 1). Overall, the dispersion-corrected B3P86 functionals appear well-suited for this mixed 5 (electrostatic and dispersion) class of biochemically inspired systems. 6

7 Table 1. Mean Absolute Deviation (MAD, kcal.mol ${ }^{-1}$ ) on S66 subsets (i.e., Dispersion and 8 Mixed, see text) and NCCE31 subsets (i.e., NCCE31-CNOH, rare gas NCCE31-RG and halogen 9 NCCE31-X, see text) for selected functionals of interest with or without dispersion corrections.

10 All the calculations were performed with the Def2-QZVPP basis set.

\begin{tabular}{|c|c|c|c|c|c|c|}
\hline \multirow{2}{*}{ Functional } & \multirow{2}{*}{ Dispersion correction } & \multicolumn{2}{|l|}{ S66 } & \multicolumn{3}{|l|}{ NCCE31 } \\
\hline & & Dispersion & Mixed & CNOSH & RG & $\mathrm{X}$ \\
\hline \multirow{3}{*}{ BLYP } & Uncorrected & 6.08 & 3.72 & 2.39 & 0.39 & 1.25 \\
\hline & $\mathrm{D} 3(\mathrm{BJ})$ & 0.20 & 0.11 & 0.30 & 0.13 & 2.02 \\
\hline & D3(ZD) & 0.52 & 0.32 & 0.41 & 0.07 & 1.82 \\
\hline \multirow{3}{*}{ BP86 } & Uncorrected & 5.50 & 3.31 & 2.02 & 0.60 & 1.28 \\
\hline & $\mathrm{D} 3(\mathrm{BJ})$ & 0.36 & 0.18 & 0.42 & 0.36 & 2.08 \\
\hline & D3(ZD) & 0.70 & 0.46 & 0.52 & 0.29 & 2.02 \\
\hline \multirow{3}{*}{ B3LYP } & Uncorrected & 5.11 & 2.96 & 1.72 & 0.29 & 0.64 \\
\hline & $\mathrm{D} 3(\mathrm{BJ})$ & 0.18 & 0.15 & 0.39 & 0.07 & 1.16 \\
\hline & D3(ZD) & 0.29 & 0.23 & 0.34 & 0.03 & 0.99 \\
\hline \multirow{5}{*}{ B3P86 } & Uncorrected & 4.70 & 2.69 & 1.48 & 0.46 & 0.66 \\
\hline & D2 & 0.66 & 0.26 & 0.58 & 0.24 & 0.88 \\
\hline & $\mathrm{D} 3(\mathrm{BJ})$ & 0.15 & 0.14 & 0.42 & 0.27 & 1.01 \\
\hline & D3(ZD) & 0.15 & 0.14 & 0.36 & 0.23 & 0.91 \\
\hline & $\mathrm{NL}(\mathrm{b}=5.1)$ & 0.17 & 0.18 & 0.56 & 0.21 & 1.43 \\
\hline
\end{tabular}

\section{3.2. The Hydrogen bonding HB23 database}

13 The HB23 database is an improved version of the S66 electrostatic-like subset, in which 14 association energies have been recently refined by Di Labio et al [43]. Indeed, the original S66 15 database possibly underestimated association energies of the electrostatic-like subset systems due 16 to the counterpoise correction used originally. 
As it should be, B3P86 dispersion corrected MAD values are significantly lower than those from the uncorrected B3P86. B3P86-D3(BJ) exhibits the lowest MAD (MAD $=0.40,0.42,0.43$ and $0.70 \mathrm{kcal}^{\mathrm{mol}}{ }^{-1}$ for B3P86-D3(BJ), B3P86-D3(ZD), B3P86-D2 and B3P86-NL, respectively - see Figure 2b). No significant differences were observed between the DFT-D2 and DFT-D3 corrections. A slightly higher MAD was observed for B3P86-NL; however, this functional should not be discarded since MAD is still acceptably low.

In summary, B3P86-D3(BJ) exhibits reliable results with respect to the best performing BLYP-D3(BJ) $\left(\mathrm{MAD}=0.22\right.$ and $0.40 \mathrm{kcal}^{\mathrm{mol}}{ }^{-1}$ for BLYP-D3(BJ) and B3P86-D3(BJ), respectively, Figure 2b).

\subsection{The atypical long-range NCCE31 database}

The S66 database focuses on biochemically inspired systems, so only compounds made of carbon, nitrogen, oxygen and hydrogen are included, as being the most common atoms of living organisms. However, dispersion effects attributed to halogen atoms, as chlorine and fluorine, may exhibit some specific behavior due to larger polarizability effects. To explore further this feature, the NCCE31 database was built by Truhlar et al. [44-46], in which chemical systems include either halogen atoms, namely chlorine and fluorine, or rare gas atoms, namely helium, neon and argon.

The results from B3P86-D3(ZD) closely agree with those from B3LYP-D3(ZD). Indeed, these two dispersion-corrected hybrid functionals provided the best description of long-range interactions on such systems (MAD of 0.55 and $0.53 \mathrm{kcal}^{\mathrm{mol}}{ }^{-1}$, respectively, see Figure 2c). This highlights the importance of HF-like exchange to improve description of systems containing halogen atoms. However, MAD values were significantly higher than those obtained here for the S66 and HB23 databases. As a representative example, B3P86-D3(BJ) MAD was 0.62 and 0.24 kcal.mol ${ }^{-1}$ for NCCE31 and S66, respectively (see Figure 2a\&c). Nevertheless, all dispersion-

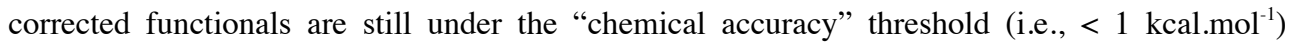

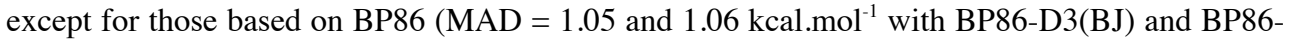
D3(ZD), respectively, see Figure 2c).

The lower performance on NCCE31 was thoroughly studied by designing three ad hoc NCCE31 subsets according to atomic composition, namely (i) NCCE31-CNOSH containing exclusively C-, N-, O-, S- and H-atoms; (ii) NCCE31-RG containing helium, neon, argon and
Florent Di Meo 21/9/2015 13:25

Deleted: exhibits the best performance ( Florent Di Meo 21/9/2015 13:25

Deleted: , being in the same range than B3P86D3(BJ), BP86-D3(BJ) and B3LYP-D3(BJ) (e.g., $\mathrm{MAD}=0.40,0.42$ and $0.37 \mathrm{kcal} . \mathrm{mol}^{-1}$, respectively, 
without halogen; and (iii) NCCE31-X containing chlorine and fluorine but discarding rare gas atoms.

We note first that all dispersion-corrected functionals provided excellent results for both NCCE31-CNOSH and NCCE31-RG subsets (see Table 1) in which dispersion has a major contribution. For instance, B3P86-D3(BJ) exhibits MAD values lower than $0.45 \mathrm{kcal}^{\mathrm{mol}}{ }^{-1}(0.42$ and $0.27 \mathrm{kcal} . \mathrm{mol}^{-1}$ for NCCE31-CNOSH and NCCE31-RG-based subsets, respectively, Table $1)$.

On the other hand, the dispersion-corrected functionals failed at accurately describing the NCCE31-X subset, providing deviations even higher than the corresponding uncorrected forms (Table 1). The presence of halogens in such small systems increases the local electrostatic bond feature, which is inherently well described by uncorrected functionals. Therefore, the use of such dispersion corrections tends to overbind NCCE31-X complexes by overestimating the dispersion contribution, Mean Deviation being e.g., -0.81 and $-0.48 \mathrm{kcal}^{-\mathrm{mol}^{-1}}$ for B3LYP-D3(BJ) and B3P86-D3, respectively (see Supporting Information).

\section{Assessment of the B3P86-D3 model for large systems}

\subsection{The S12L database}

Recently, the S12L database has been proposed to assess dispersion-corrected functionals on large $\pi$-delocalized systems having real-world applications, including e.g., non-covalent complexes with $\mathrm{C}_{60}$ and $\mathrm{C}_{70}$ fullerenes or dicationic ferrocene derivatives [47]. This database has been especially designed to assess both the three-body term (so-called ABC-term) contained in the DFT-D3 formalism, as well as the thermodynamic contributions. It must be stressed that the present study only deals with the former; vibrational analysis would have required huge computational resources, which are beyond the scope of work. Note also that calculations were performed here with the triple- $\zeta$ basis set due to some numerical instabilities when using the quadruple- $\zeta$ basis set. This is however not expected to largely influence the conclusions dropped here.

The different dispersion corrections are able to drastically improve the description of S12L association energies (see Table 2). Interestingly, B3P86-D2 provides a very low error (MAD = $1.6 \mathrm{kcal} . \mathrm{mol}^{-1}$ ) while B3P86-NL appears not so suited to predict association energies for these 
1 systems $\left(\mathrm{MAD}=8.3 \mathrm{kcal}^{\mathrm{mol}}{ }^{-1}\right) . \mathrm{B} 3 \mathrm{P} 86-\mathrm{NL}$ should thus be carefully used for the large S12L 2 systems. DFT-D3 correction ranges from 2.2 to $6.0 \mathrm{kcal}^{\mathrm{mol}} \mathrm{m}^{-1}$ (see Table 2), the relevance of the

3 ABC-term within the DFT-D3 framework was previously highlighted for such big systems [47].

4 DFT-D3(BJ) is more affected by ABC-term correction than DFT-D3(ZD), the MAD difference 5 between the uncorrected and corrected values being 2.2 and $1.0 \mathrm{kcal}^{\mathrm{mol}}{ }^{-1}$ for DFT-D3(BJ) and 6 DFT-D3(ZD), respectively (Table 2).

8 Table 2. Association energies $\left(\mathrm{kcal}_{\mathrm{mol}}{ }^{-1}\right)$ and mean absolute deviation (MAD, $\left.\mathrm{kcal} . \mathrm{mol}^{-1}\right)$ for 9 S12L dataset. All the calculations were performed with the Def2-TZVPP basis set.

\begin{tabular}{|c|c|c|c|c|c|c|c|c|}
\hline \multirow{2}{*}{ Structure } & \multirow{2}{*}{ B3P86 } & \multirow{2}{*}{$\begin{array}{l}\text { B3P86 } \\
\text {-D2 }\end{array}$} & \multicolumn{2}{|c|}{ B3P86-D3(BJ) } & \multicolumn{2}{|c|}{ B3P86-D3(ZD) } & \multirow{2}{*}{$\begin{array}{l}\text { B3P86 } \\
-\mathrm{NL}^{a}\end{array}$} & \multirow{2}{*}{ Reference $^{t}$} \\
\hline & & & no-ABC & $\mathrm{ABC}$ & no-ABC & $\mathrm{ABC}$ & & \\
\hline 1 & 2.1 & -1.6 & -3.1 & -3.0 & -2.5 & -2.4 & -2.6 & -2.8 \\
\hline $2 \mathrm{a}$ & 1.6 & -29.7 & -37.1 & -35.2 & -32.2 & -30.4 & -37.0 & -30.3 \\
\hline $2 b$ & 3.4 & -19.5 & -24.7 & -23.3 & -20.9 & -19.6 & -24.2 & -20.5 \\
\hline $3 a$ & 2.1 & -23.1 & -29.6 & -27.7 & -27.0 & -25.2 & -32.3 & -24.4 \\
\hline $3 b$ & -5.4 & -18.7 & -22.2 & -21.5 & -21.3 & -20.5 & -24.5 & -20.4 \\
\hline $4 a$ & 14.9 & -30.9 & -41.1 & -37.7 & -30.4 & -27.2 & -44.9 & -31.8 \\
\hline $4 b$ & 15.4 & -33.1 & -44.3 & -40.5 & -33.2 & -29.6 & -47.0 & -32.6 \\
\hline $5 a$ & -14.8 & -36.7 & -38.7 & -37.6 & -38.2 & -37.2 & -41.5 & -31.9 \\
\hline $5 b$ & -3.9 & -23.1 & -26.3 & -25.2 & -25.2 & -24.2 & -28.1 & -20.4 \\
\hline $6 a$ & -61.9 & -83.0 & -87.7 & -85.3 & -87.6 & -85.3 & -89.9 & -82.2 \\
\hline $6 b$ & -62.0 & -79.8 & -83.7 & -81.7 & -83.5 & -81.6 & -85.1 & -78.9 \\
\hline $7 \mathrm{a}$ & -84.4 & -132.9 & -138.7 & -133.8 & -137.2 & -132.3 & -146.0 & -129.6 \\
\hline $7 b$ & 5.0 & -25.2 & -31.7 & -28.2 & -30.2 & -26.8 & -35.5 & -25.7 \\
\hline$M A D$ & 26.4 & 1.6 & 6.0 & 3.8 & 3.2 & 2.2 & 8.3 & - \\
\hline
\end{tabular}


We are aware that these results can be analyzed only at a semi-quantitative level. The basis set used is not sufficiently large and the use of counterpoise (CP) correction might overestimate the associated BSSE [47]. However, some trends emerged from the array of data [54,55]. As a matter of illustration, the D3-like corrections used led to an accurate description of these systems considering that the "chemical accuracy" (defined as an error below $1 \mathrm{kcal}^{\mathrm{mol}}{ }^{-1}$ ) is unrealistic for large systems. In other words, an error of 2-3 $\mathrm{kcal}_{\mathrm{mol}}{ }^{-1}$ would be a more realistic target.

The common dispersion correction tends to overestimate association energies (see Supporting Information) and, finally, the use of the repulsive ABC-term for B3P86-D3 significantly decreases this overestimation.

\subsection{Natural polyphenol dataset}

Over the past years, the chemistry of polyphenol has been of utmost importance in food science. Non-covalent interactions have been shown to play a key role in optical, chemical and biological properties of polyphenols $[17,18,15,12,16,22]$. Even if we are aware that theoretical chemistry aims at finding the generally applicable methods, we believe that, regarding the huge amount of phenolic derivatives and the huge amount of possible non-covalent association of interest, both dispersion and H-bonding interactions drive polyphenol non-covalent dimerization. Thus, we still believe that this family deserves particular attention (i.e. specific methods and database for further benchmarking).

Therefore, the seven non-covalent polyphenol systems studied here include the phenol Tshaped dimer taken from S22; for which the geometry was obtained at the MP2/CBS level of theory [29]. This system is driven only by H-bond since no $\pi$-stacked conformation has been identified yet as being more stable.

The complete potential energy surface of the 3-O-methylcyanidin:quercetin dimer (namely C:Q) was previously explored at the B3P86-D2/cc-pVDZ level of theory, revealing the existence of five conformations, namely $\mathbf{C : Q}$ conformers 1-5 [15]. These systems are mainly driven by $\pi$ stacking forces, as favored by the strong $\pi$-electron delocalization character of $\mathbf{C}: \mathbf{Q}$. The existence of numerous $\mathrm{OH}$ groups in each partner (see Scheme 1) induces a strong competition between intra- and intermolecular H-bonding (Figure 1), making the global H-bonding 
(a)

\begin{tabular}{|c|c|c|c|c|c|c|c|c|c|}
\hline & \multirow[b]{2}{*}{ Conf } & \multirow[b]{2}{*}{ В3Р86 } & \multirow{2}{*}{$\begin{array}{l}\text { B3P86 } \\
\text {-D2 }\end{array}$} & \multicolumn{2}{|c|}{ B3P86-D3(BJ) } & \multicolumn{2}{|c|}{ B3P86-D3(ZD) } & \multicolumn{2}{|c|}{ B3P86-NL } \\
\hline & & & & $\begin{array}{l}\text { no- } \\
\text { ABC }\end{array}$ & $\mathrm{ABC}$ & $\begin{array}{l}\text { no- } \\
\text { ABC }\end{array}$ & $\mathrm{ABC}$ & $b=5.1$ & $b=5.3$ \\
\hline Phenol dimer & & -3.7 & -6.9 & -7.3 & -7.2 & -7.3 & -7.3 & -7.5 & -7.3 \\
\hline \multirow[t]{5}{*}{$\mathrm{C}: \mathbf{Q}$} & 1 & 14.0 & -9.8 & -12.8 & -11.6 & $\begin{array}{l}-10.3 \\
\end{array}$ & -9.2 & $\begin{array}{l}-14.7 \\
\end{array}$ & -13.5 \\
\hline & 2 & 14.5 & -10.4 & -13.1 & -11.9 & -11.0 & -9.8 & -15.3 & -14.0 \\
\hline & 3 & 14.8 & -11.2 & -15.1 & -13.8 & -12.1 & -10.8 & -17.1 & -15.8 \\
\hline & 4 & 14.9 & -10.5 & -13.8 & -12.5 & -11.5 & -10.3 & -15.8 & -14.5 \\
\hline & 5 & 16.8 & -10.9 & -14.0 & -12.6 & -11.5 & -10.1 & -16.7 & -15.2 \\
\hline$\varepsilon$-viniferin dim & & 21.5 & -8.5 & -11.6 & -9.8 & -9.5 & -7.8 & -14.3 & -12.9 \\
\hline
\end{tabular}

reasonable conformational sampling. The Boltzmann-weighted average association energy derived from the relative electronic energy between all five conformers can then be compared to the reference experimental value [52].

The third system includes the $\varepsilon$-viniferin dimer previously elucidated [14]. This system exhibits both $\mathrm{H}$-bonding and $\pi$-stacking contributions. The complete potential energy surface has not been yet explored due to the high number of degrees of freedom and the system size, however this dimer might be used as a relevant system for studying the interplay between hybrid H-bonding and $\pi$-stacking.

Table 3. BSSE-uncorrected association energies $\left(\mathrm{kcal} \cdot \mathrm{mol}^{-1}\right)$ for the 7-member group of noncovalent natural polyphenol systems with (a) B3P86 and (b) SCS-MP2 methods refinements. All the calculations were performed with Def2-TZVPP basis set.

(b)

\begin{tabular}{lllll}
\hline & Conf. & SCS-MP2 & SCS-MP2- D2 & SCS-S66-MP2 \\
\hline Phenol dimer & & -6.9 & -7.5 & -8.0 \\
\hline $\mathbf{C : Q}$ & $\mathbf{1}$ & -15.8 & -20.7 & -21.7 \\
& $\mathbf{2}$ & -16.4 & -21.5 & -24.8 \\
& $\mathbf{3}$ & -20.1 & -25.4 & -24.8 \\
& $\mathbf{4}$ & -18.0 & -23.2 & -22.8
\end{tabular}




\begin{tabular}{lllll} 
& 5 & -18.8 & -24.5 & -22.9 \\
\hline$\varepsilon$-viniferin dimer & & -20.3 & -26.4 & -14.2 \\
\hline
\end{tabular}

2

Assessment of association energies. For the phenol dimer, the reference association energy $(\Delta \mathrm{E}=$ -7.1 kcal.mol ${ }^{-1}$, see Figure 3a) was obtained with the current computationally acceptable "gold standard" CCSD(T)/CBS level of theory [38]. The uncorrected B3P86 method exhibits association energy of $-3.7 \mathrm{kcal}^{\mathrm{mol}}{ }^{-1}$, pointing out at least the qualitative correct description of $\mathrm{H}$-bonds (electrostatic-like) by B3P86. However, the use of dispersion correction is mandatory for a more complete description of all the non-covalent interactions arising in the phenol dimer. All dispersion corrected functionals (i.e., DFT-D or DFT-NL) provided association energies in the range from -7.5 to $-6.9 \mathrm{kcal} . \mathrm{mol}^{-1}$, and thus in very good agreement with the CCSD(T)/CBS reference value (see Table 3 and Figure $3 a$ ). The triple- $\zeta$ basis set seems to be sufficient to reach up the basis set limit for such a small system. The use of the ABC-term does not bring any significant difference, as it was previously suggested for small systems $[28,47]$. It must be stressed that two slightly different forms of B3P86-NL were assessed here. Recently, a different attenuating parameter $b=5.3$ [57] was proposed to couple the $-\mathrm{NL}$ correction to the B3P86 functional, comparable to our previous estimate of $b=5.1$ [14]. As it is expected, association energies do not differ between both methods (i.e., $\Delta \mathrm{E}=-7.5$ and $-7.3 \mathrm{kcal}_{\mathrm{mol}}{ }^{-1}$, for $b=5.1$ and 5.3, respectively, see Table 3 and Figure 3a).

The association energy of reference for $\mathbf{C}: \mathbf{Q}$ complexes was experimentally derived in solution $\left(\Delta \mathrm{H}=-13.9 \mathrm{kcal}_{\mathrm{mol}}{ }^{-1}\right.$, see Table 4 and Figure $\left.3 \mathrm{~b}\right)$ [52]. It must be stressed that calculations from the present study do not include thermodynamic corrections. However, no significant differences between enthalpy and internal energies are expected: vibrational thermal corrections of each free partner are expected to cancel the global vibrational thermal correction on the dimer. It must be stressed that specific solvation contributions to enthalpy are neglected. Theoretical association energies are either presented for each conformation (Table 3) or as a Boltzmannweighted interaction energy $\left(\Delta \mathrm{E}_{\text {boltz }}\right.$, see Table 4 and Figure $\left.3 b\right)$. The latter is used when theoretical results are compared to the experimental value.

B3P86-D2 association energies in the present study are significantly higher (by $2.8 \mathrm{kcal}^{\mathrm{mol}}{ }^{-1}$ ) than those performed in our previous study [15] (see Table 3). Our previous association energies 
1 were performed with a lower basis set and corrected by the counterpoise correction, and will be 2 thus recalculated here. DFT-D3 is the best performing with respect to DFT-NL and DFT-D2, 3 providing the closest value to experiment (Table 4 and Figure $3 b$ ). The use of the three-body 4 term together with the ZD-damping function obviously enhances the error by adding the ABCrepulsive term $\left(\Delta \mathrm{E}_{\mathrm{boltz}}=-11.8\right.$ and $-10.5 \mathrm{kcal}^{\mathrm{mol}}{ }^{-1}$ for $\mathrm{B} 3 \mathrm{P} 86-\mathrm{D} 3(\mathrm{ZD})$ without or with threebody correction, respectively, Table 4). The use of the BJ-damping function is thus recommended to describe such systems since results are closer to the experimental value $\left(\Delta \mathrm{E}_{\mathrm{boltz}}\right.$ $8=-13.4$ and $-14.8 \mathrm{kcal}^{\mathrm{mol}} \mathrm{m}^{-1}$ for B3P86-D3(BJ) with or without three-body correction, 9 respectively, Table 4). C:Q are systems big enough to be significantly affected by the three-body correction, which is thus recommended to be always included for large supramolecular complexes.

Table 4. Calculated and reference association energies $\left(\Delta \mathrm{E}, \mathrm{kcal}_{\mathrm{mol}}{ }^{-1}\right)$ of Boltzmann-weighted C:Q subset. All the calculations were performed with the Def2-TZVPP basis set.

\begin{tabular}{lllc}
\hline Method & & & $\Delta \mathrm{E}_{\text {boltz }}{ }^{a}$ \\
\hline DFT & B3P86 & & 14.3 \\
& B3P86-D2 & & -10.9 \\
& B3P86-D3(BJ) & No-ABC & -14.8 \\
& & ABC & -13.4 \\
& B3P86-D3(ZD) & No-ABC & -11.8 \\
& & ABC & -10.5 \\
& B3P86-NL & $\mathrm{b}=5.1$ & -16.8 \\
& B3P86-NL(b=5.3) & $\mathrm{b}=5.3$ & -15.5 \\
\hline SCS-MP2 & SCS-MP2 & & -19.9 \\
& SCS-MP2-D2 & & -25.2 \\
& SCS-S66-MP2 & & -24.7 \\
\hline
\end{tabular}

${ }^{a}$ Experiments exhibits association enthalpy $\Delta H=-13.9$ kcal.mol ${ }^{-1}$, see Ref. [52]

Regarding association energies of the $\varepsilon$-viniferin, the following sequence is observed: B3P86D2 $\approx$ B3P86-D3(ZD)-ABC > B3P86-D3(ZD) > B3P86-D3(BJ)-ABC > B3P86-D3(BJ) > B3P86NL. It must be stressed that neither experimental reference value nor high-level post-HF calculations (i.e., CCSD(T)/CBS calculation) are available for this dimer. The association energy of $\varepsilon$-viniferin dimer was previously obtained at the SCS-MP2/Def2-TZVPP level of theory, 
1 which will be confirmed here [14]. It must be stressed that SCS-MP2 method consists in a 2 refinement of MP2 one, in which spin-paired and spin-unpaired electron contributions are scaled 3 [58]. Three SCS-MP2 methods were actually also assessed for the 7 non-covalent polyphenol 4 prototypes, namely the native SCS-MP2 method [58] and a pair of dispersion-driven 5 refinements; the SCS-MP2-D2; and the SCS-S66-MP2 variant [59]. Indeed, its native form 6 (SCS-MP2) exhibits significant error with respect to S66, while the SCS-MP2-D2 refinements 7 for non-covalent interactions are expected to be more accurate for this study [42,59].

8 For the sake of comparison, the BLYP, BP86 and B3LYP functionals (with and without their 9 respective DFT-D3 correction) were also assessed and their results reported in Figure 3. 

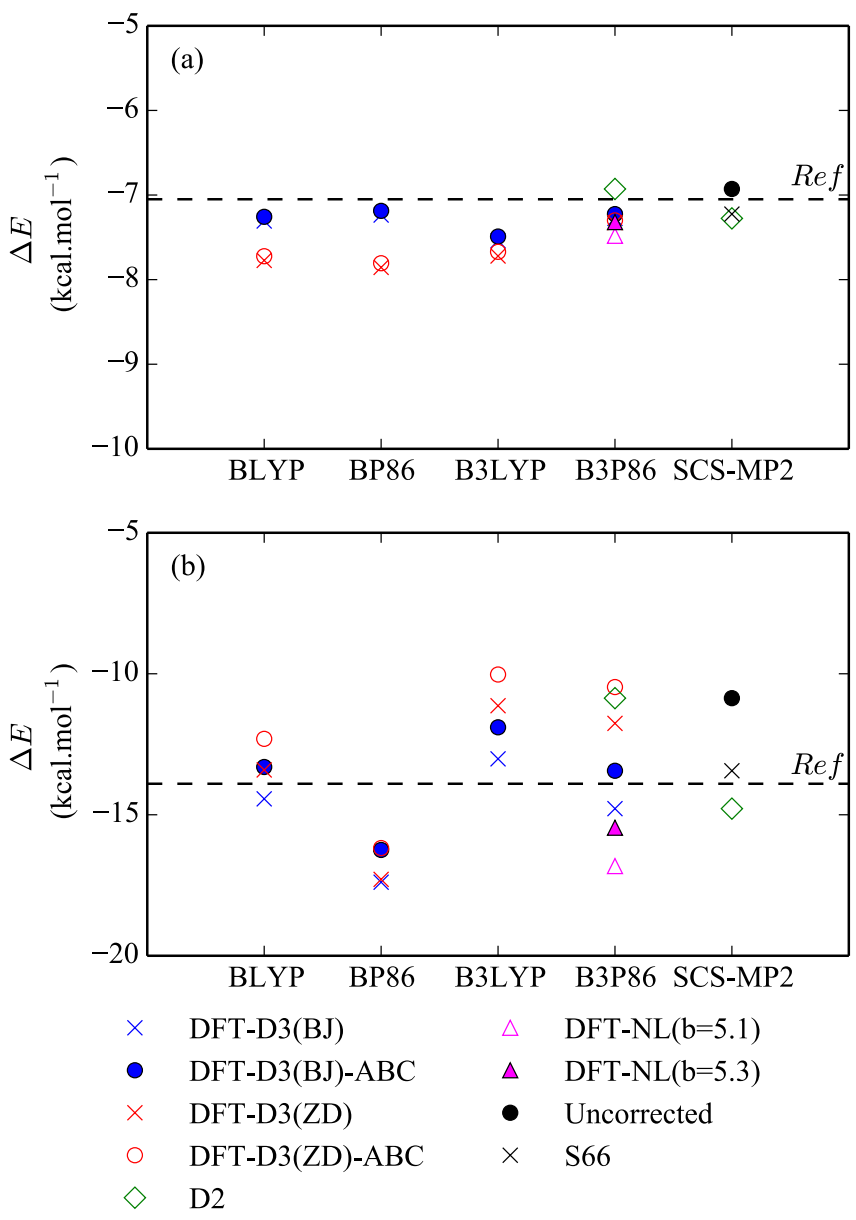

2 Figure 3. Calculated and reference association energies $\left(\Delta \mathrm{E}, \mathrm{kcal} . \mathrm{mol}^{-1}\right)$ of (a) Phenol dimer and

3 (b) Boltzmann-weighted C:Q subset. All the calculations were performed with the Def2-TZVPP 4 basis set.

5 It must be noticed that all SCS-MP2 based methods overestimate binding of phenol dimer and $6 \mathbf{C : Q}$ complexes with respect to reference values (see Table $3 b$ and Figure 3 ). The use of SCS-

7 MP2 related methods is therefore not as appropriate as dispersion-corrected DFT, considering 8 the involved higher computational effort. The same concluding remark could be applied to 9 B3P86-NL, but to a lesser extent. This formalism tends to globally overestimate binding for the 
7-member group of non-covalent natural polyphenol systems as shown in Tables 4 and Figure 3. Interestingly, among conventional DFT functionals, BLYP and B3LYP exhibit good agreement with respect to reference only with DFT-D3(BJ) correction including ABC-term while BP86 tends to overestimate polyphenol binding energies. Such results again highlight the importance of three-body term for polyphenol systems since only DFT-D3 corrections including ABC term provide acceptable results. Among all dispersion-corrected DFT assessed here, the following sequence is given BP86-D3(BJ) < B3P86-NL $\approx$ B3P86-D2 < B3LYP-D3(BJ) < BLYP-D3(BJ) $\approx$ B3P86-D3(BJ), in terms of accuracy.

\section{Conclusion}

In this study, the parameterization of the B3P86-D3 dispersion-corrected model is done against the S66, and further assessed using the HB23, NCCE31 and S12L databases of non-covalent compounds. Both the BJ- and ZD-damping functions were systematically evaluated. The performance of B3P86-D3 has been compared with that of BLYP-D3, BP86-D3 and B3LYP-D3, showing that B3P86-D3(BJ) emerges one of the best performing and cost-efficient methods for real-world phenolic systems. We have also confirmed the goof performance of B3P86-D3 on the 7-member group of non-covalent natural polyphenol systems providing a significant improvement with respect to the other previously derived dispersion-corrected B3P86 methods. B3P86-D3(BJ) is thus recommended for dealing with challenging (supramolecular issues) in polyphenol chemistry, also considering the low computational effort compare to the "goldstandard" $\operatorname{CCSD}(\mathrm{T})$ or related SCS-MP2 methods. Keeping in mind that environmental conditions (e.g., polarity, pH, specific intermolecular interaction with solvent, presence of metal ions) should also be carefully treated when needed, we believe that the methodology is fully applicable for i) food and wine application (tuning optical properties in copigmentation complexes), and ii) in biology (antioxidant) properties.

Supporting Information. Description of non-covalent refinement within DFT formalism; DFTD3 parameters for studied functionals; Interaction energies for studied functionals with S66, HB23 and NCCE31 databases; Global statistical analysis including MAD, RMSD and MD for S66, HB23, NCCE31, and S12L databases; XYZ-coordinates of seven non-covalent polyphenol 
1 systems and corresponding stand-alone polyphenols; Assessment of RIJCOSX reliability to energy minimum for $\mathbf{C}: \mathbf{Q}$ system

\section{ACKNOWLEDGMENT}

4

The authors acknowledge computational support from "CALI" (“CAlcul en LImousin”). FDM acknowledges financial support from the Swedish Research Council (Grant No. 621-2014-4646) and SNIC (Swedish National Infrastructure for Computing) for providing computer resources. The work in Limoges (IB and PT) is supported by the "Conseil Régional du Limousin". PT gratefully acknowledges the support by the Operational Program Research and Development Fund (project CZ.1.05/2.1.00/03.0058 of the Ministry of Education, Youth and Sports of the Czech Republic). IB gratefully acknowledges financial support from "Association Djerbienne en France".

\section{REFERENCES}

1. Rice-Evans CA, Packer L (1998) Flavonoids in Health and Disease. vol Copyright (C) 2012 American Chemical Society (ACS). All Rights Reserved. Dekker, New York

2. Manach C, Scalbert A, Morand C, Rémésy C, Jiménez L (2004) Polyphenols: food sources and bioavailability. Am J Clin Nutr 79:727-747

3. Hertog MG, Feskens EJ, Hollman PC, Katan MB, Kromhout D (1993) Dietary antioxidant flavonoids and risk of coronary heart disease: the Zutphen Elderly Study. Lancet 342:1007-1011

4. Saller R, Meier R, Brignoli R (2001) The use of silymarin in the treatment of liver diseases. Drugs 61:2035-2063

5. Gazak R, Marhol P, Purchartova K, Monti D, Biedermann D, Riva S, Cvak L, Kren V (2010) Largescale separation of silybin diastereoisomers using lipases. Process Biochem 45:1657-1663 6. Commenges D, Scotet V, Renaud S, Jacqmin-Gadda H, Barberger-Gateau P, Dartigues JF (2000) Intake of flavonoids and risk of dementia. Eur J Epidemiol 16:357-363

7. Snyder SA, Gollner A, Chiriac MI (2011) Regioselective reactions for programmable resveratrol oligomer synthesis. Nature 474:461-466

8. Leopoldini M, Marino T, Russo N, Toscano M (2004) Antioxidant Properties of Phenolic Compounds: H-Atom versus Electron Transfer Mechanism. J Phys Chem A 108:4916-4922

9. Leopoldini M, Marino T, Russo N, Toscano M (2004) Density functional computations of the energetic and spectroscopic parameters of quercetin and its radicals in the gas phase and in solvent. Theor Chem Acc 111:210-216 
10. Chiodo SG, Leopoldini M, Russo N, Toscano M (2010) The inactivation of lipid peroxide radical by

2 quercetin. A theoretical insight. Phys Chem Chem Phys 12:7662-7670

3 11. Quartarolo AD, Russo N (2011) A Computational Study (TDDFT and RICC2) of the Electronic

4 Spectra of Pyranoanthocyanins in the Gas Phase and Solution. J Chem Theory Comput 7:1073-1081

5 12. Di Meo F, Lemaur V, Cornil J, Lazzaroni R, Duroux J-L, Olivier Y, Trouillas P (2013) Free Radical

6 Scavenging by Natural Polyphenols: Atom versus Electron Transfer. J Phys Chem A 117:2082-2092

7 13. Rustioni L, Di Meo F, Guillaume M, Failla O, Trouillas P (2013) Tuning color variation in grape

8 anthocyanins at the molecular scale. Food Chem 141:4349-4357

9 14. Bayach I, Sancho-García JC, Di Meo F, Weber JFF, Trouillas P (2013) $\pi$-Stacked polyphenolic

10 dimers: A case study using dispersion-corrected methods. Chem Phys Lett 578:120-125

15. Di Meo F, Sancho García JC, Dangles O, Trouillas P (2012) Highlights on Anthocyanin Pigmentation and Copigmentation: A Matter of Flavonoid $\pi$-Stacking Complexation To Be Described by DFT-D. J Chem Theory Comput 8:2034-2043

16. Boulton R (2001) The Copigmentation of Anthocyanins and Its Role in the Color of Red Wine: A Critical Review. Am J Enol Vitic 52:67-87

17. Nave F, Brás NF, Cruz L, Teixeira N, Mateus N, Ramos MJ, Di Meo F, Trouillas P, Dangles O, De Freitas V (2012) Influence of a Flavan-3-ol Substituent on the Affinity of Anthocyanins (Pigments) toward Vinylcatechin Dimers and Proanthocyanidins (Copigments). J Phys Chem B 116:14089-14099 18. Velu SS, Di Meo F, Trouillas P, Sancho García J-C, Weber J-FF (2013) Regio- and Stereocontrolled Synthesis of Oligostilbenoids: Theoretical Highlights at the Supramolecular Level. J Nat Prod 76:538-546 19. Velu SS, Thomas NF, Weber J-FF (2012) Strategies and methods for the syntheses of natural oligomeric stilbenoids and analogues. Curr Org Chem 16:605-662 20. Velu SS, Buniyamin I, Ching LK, Feroz F, Noorbatcha I, Gee LC, Awang K, Wahab IA, Weber J-FF (2008) Regio- and Stereoselective Biomimetic Synthesis of Oligostilbenoid Dimers from Resveratrol Analogues: Influence of the Solvent, Oxidant, and Substitution. Chemistry - A European Journal 14:11376-11384

21. Cheynier V (2012) Phenolic compounds: From plants to foods. Phytochem Rev 11:153-177 Interactions between Deoxyanthocyanidin Derivatives (Oaklins) and Common Copigments in Wine Model Solutions. J Agric Food Chem significance. Curr Org Chem 16:692-714 
1 24. Anouar EH, Raweh S, Bayach I, Taha M, Baharudin MS, Di Meo F, Hasan MH, Adam A, Ismail NH,

2 Weber JFF, Trouillas P (2013) Antioxidant properties of phenolic Schiff bases: Structure-activity

3 relationship and mechanism of action. J Comput Aided Mol Des 27:951-964

425 . Grimme S (2004) Accurate description of van der Waals complexes by density functional theory

5 including empirical corrections. J Comput Chem 25:1463-1473

6 26. Grimme S (2006) Semiempirical GGA-type density functional constructed with a long-range

7 dispersion correction. J Comput Chem 27:1787-1799

8 27. Grimme S, Antony J, Ehrlich S, Krieg H (2010) A consistent and accurate ab initio parametrization of 9 density functional dispersion correction (DFT-D) for the 94 elements H-Pu. J Chem Phys 132

10 28. Grimme S, Ehrlich S, Goerigk L (2011) Effect of the damping function in dispersion corrected density functional theory. J Comput Chem 32:1456-1465

29. Gráfová L, Pitonák M, Řezác J, Hobza P (2010) Comparative Study of Selected Wave Function and Density Functional Methods for Noncovalent Interaction Energy Calculations Using the Extended S22

Data Set. J Chem Theory Comput 6:2365-2376

30. Jurečka P, Černý J, Hobza P, Salahub DR (2007) Density functional theory augmented with an

empirical dispersion term. Interaction energies and geometries of 80 noncovalent complexes compared

with ab initio quantum mechanics calculations. J Comput Chem 28:555-569

31. Wu Q, Yang W (2002) Empirical correction to density functional theory for van der Waals interactions. J Chem Phys 116:515-524 32. Dion M, Rydberg H, Schröder E, Langreth DC, Lundqvist BI (2004) Van der Waals Density Functional for General Geometries. Phys Rev Lett 92:246401 33. Vydrov OA, Van Voorhis T (2010) Nonlocal van der Waals density functional: The simpler the better. J Chem Phys 133:244103 34. Hujo W, Grimme S (2011) Performance of the van der Waals Density Functional VV10 and (hybrid)GGA Variants for Thermochemistry and Noncovalent Interactions. J Chem Theory Comput 7:3866-3871 35. Trouillas P, Fagnere C, Lazzaroni R, Calliste C, Marfak A, Duroux J-L (2004) A theoretical study of the conformational behavior and electronic structure of taxifolin correlated with the free radicalscavenging activity. Food Chem 88:571-582 36. Anouar EH, Gierschner J, Duroux J-L, Trouillas P (2012) UV/Visible spectra of natural polyphenols: A time-dependent density functional theory study. Food Chem 131:79-89

32 37. Millot M, Di Meo F, Tomasi S, Boustie J, Trouillas P (2012) Photoprotective capacities of lichen metabolites: A joint theoretical and experimental study. J Photochem Photobiol B 111:17-26 
1 38. Jurecka P, Sponer J, Cerny J, Hobza P (2006) Benchmark database of accurate (MP2 and CCSD(T)

2 complete basis set limit) interaction energies of small model complexes, DNA base pairs, and amino acid

3 pairs. Phys Chem Chem Phys 8:1985-1993

4 39. Becke AD, Johnson ER (2005) Exchange-hole dipole moment and the dispersion interaction. J Chem

5 Phys 122:154104

6 40. Johnson ER, Becke AD (2006) A post-Hartree-Fock model of intermolecular interactions: Inclusion

7 of higher-order corrections. J Chem Phys 124:174104

8 41. Johnson ER, Becke AD (2005) A post-Hartree-Fock model of intermolecular interactions. J Chem

9 Phys 123:024101

10 42. Rězác J, Riley KE, Hobza P (2011) S66: A Well-balanced Database of Benchmark Interaction

11 Energies Relevant to Biomolecular Structures. J Chem Theory Comput 7:2427-2438

12 43. DiLabio GA, Johnson ER, Otero-de-la-Roza A (2013) Performance of conventional and dispersioncorrected density-functional theory methods for hydrogen bonding interaction energies. Phys Chem Chem

14 Phys 15:12821-12828

44. Zhao Y, Truhlar DG (2005) Design of Density Functionals That Are Broadly Accurate for

Thermochemistry, Thermochemical Kinetics, and Nonbonded Interactions. J Phys Chem A 109:5656-

$17 \quad 5667$

45. Zhao Y, Truhlar DG (2005) Benchmark Databases for Nonbonded Interactions and Their Use To Test

Density Functional Theory. J Chem Theory Comput 1:415-432 46. Peverati R, Truhlar D (2014) Quest for a universal density functional: the accuracy of density functionals across a broad spectrum of databases in chemistry and physics. Phil Trans R Soc A 372:20120476

47. Grimme S (2012) Supramolecular Binding Thermodynamics by Dispersion-Corrected Density

Functional Theory. Chemistry - A European Journal 18:9955-9964 48. Neese F (2012) The ORCA program system. Wiley Interdisciplinary Reviews: Computational Molecular Science 2:73-78 49. Grimme S (2011) DFT-D3 - A dispersion correction for density functionals, Hartree-Fock and semiempirical quantum chemical methods. http://www.thch.unibonn.de/tc/index.php?section=downloads\&subsection=DFT-D3\&lang=english. Accessed May

30 23th, 2013

31 50. Neese F, Wennmohs F, Hansen A, Becker U (2009) Efficient, approximate and parallel Hartree-Fock 32 and hybrid DFT calculations. A 'chain-of-spheres' algorithm for the Hartree-Fock exchange. Chem Phys 33 356:98-109 
1 51. Neese F (2003) An improvement of the resolution of the identity approximation for the formation of

2 the Coulomb matrix. J Comput Chem 24:1740-1747

3 52. Dimitric MJM, Baranac JM, Brdaric TP (2005) Electronic and infrared vibrational analysis of

4 cyanidin-quercetin copigment complex. Spectrochim Acta A 62A:673-680

5 53. Sinnecker S, Rajendran A, Klamt A, Diedenhofen M, Neese F (2006) Calculation of Solvent Shifts on

6 Electronic g-Tensors with the Conductor-Like Screening Model (COSMO) and Its Self-Consistent

7 Generalization to Real Solvents (Direct COSMO-RS). J Phys Chem A 110:2235-2245

8 54. Risthaus T, Grimme S (2013) Benchmarking of London Dispersion-Accounting Density Functional

9 Theory Methods on Very Large Molecular Complexes. J Chem Theory Comput 9:1580-1591

10 55. Grimme S, Mück-Lichtenfeld C, Antony J (2007) Noncovalent Interactions between Graphene Sheets

11 and in Multishell (Hyper)Fullerenes. J Phys Chem C 111:11199-11207

12 56. The Boltzmann weights are derived from the relative electronic energy between all five conformers.

13 57. Martin JML (2013) What Can We Learn about Dispersion from the Conformer Surface of n-Pentane?

14 J Phys Chem A 117:3118-3132

15 58. Grimme S (2003) Improved second-order M[o-slash]ller--Plesset perturbation theory by separate

16 scaling of parallel- and antiparallel-spin pair correlation energies. J Chem Phys 118:9095-9102

17 59. Riley KE, Platts JA, Řezáč J, Hobza P, Hill JG (2012) Assessment of the Performance of MP2 and

18 MP2 Variants for the Treatment of Noncovalent Interactions. J Phys Chem A 116:4159-4169 